\title{
GEMMEIZA 11 - A NEW EGYPTIAN HIGH YIELDING BREAD WHEAT (Triticum aestivum L.) CULTIVAR
}

Sadek, Eman M.; A. Ageez; M.El-Menofy; M. Abo Shereef; A. Hamada; A.Moussa; R. Kumber; S. Sleem; G. El- Shaarawy; A. A. morad; S. Abdel- Majeed; A. Abo- Warda; A.Tammam; M. Mesherf; E.A.M.El-Sayed; H. Ashoush; M. Toweefles; H. Hendawy;Hayam S. Mahgoub; A.K. Mostafa; H. El-Borhamy; A. Menshawy; Wafaa M. El- Awady Nadia Abd El-Nour; S. AbdelDayem; Sohair M. Hassan; A. Swelam; S. El- Sawy; S. Hamad;Magda A. Abdel - Rahman; Sabah. Abo El-Ela; M.A. Khaled; R.A. Ramadan; I. A. Amin; M. Zakaria; Manal A. Hassan; A. Gad-Allah; M. A. El-Maghraby; Aza M. Abdel-Al; A. Hagras; A.T. Mostafa; M.S. Mahmoud; M.Y.Moubark; Thanaa Abd-ElKreem; A.M.Morsy; Hoda El-Gharabawy; AglanM.A.A; W. Farahat; Abd El-Hamid E.A.M;Ragab K.I.Gad;E; Nathan Shereen;AbdelLatif, I.S.M; Abdel Kader,M.N; A. Gomaa ;Enayat Ghanem; S.Kh. Mahmoud; M.G.Mosaad; N.Hanna; M.A. Moussa; M.A.Gouda; Mostafa, M. A.; M.S. Sharshar; T. Shehab EI Din; M. Abde-Aleem; S.R.Sabry; M.A. Salem; A. Khattab; A. Abdel-Latif;A. El-Hag; Najwa Abdel- Fattah; F. Hefnawy ; ${ }^{\star}$ I. A. Imbaby and *M.M El- Shamy.

Wheat Res. Dept. Field Crops Res. Inst., ARC, Giza 12619, Egypt.

*Wheat Disease Dept., Plant Pathology Res. Inst., ARC, Giza 12619, Egypt.

\footnotetext{
ABSTRACT

The newly bread wheat cultivar Gemmeiza 11 has been selected from a local cross made at Gemmeiza Agricultural Research Station, Wheat Research Dept. FCRI, ARC. Egypt, using the pedigree method.

Yield evaluation was performed through 10 preliminary yield trials in 2005/ 2006, 65 advanced yield trials in 2006/2007 and 2007/2008 growing seasons, 66 verification yield trials in $2009 / 2010,2010 / 2011$ and $2011 / 2012$ seasons in addition to 106 demonstration fields in 2011/ 2012 growing season. Results proved the superiority of the new bread wheat cultivar Gemmeiza11 compared to the commercial check cultivars Sakha 93, Sakha 94, Gemmeiza 9 and Shandaweel 1 in the most of different zones with a few exceptions. However, at the level of over all mean of the check cultivars across the locations at different climatic zones, the new cultivar mean yield exceeded significantly all the check cultivars means in both 2006/2007 and $2007 / 2008$ growing seasons. Thus, we can highly recommend the new cultivar Gemmeiza 11 to be grown all over zones of Egypt. Moreover, the results of the verification yield trials ensure the superiority of Gemmeiza11 on the commercial wheat cultivars Sakha 93, Giza 168 and Gemmeiza 9 at North, Middle and South Delta, but at Middle Egypt its yield was less than the check cultivars Sakha 93 and Giza 168 and it was nearly equal at Upper Egypt. The obtained results of the new cultivar in the demonstration fields achieved 28.38 ard /fed in four governorates in Middle and South Delta compared to the mean of the five check cultivars which yielded 24.162 ard / fed with an increment of 4.128 ard / fed (17.46\%).
} 


\section{INTRODUCTION}

Wheat in Egypt is considered as the main winter cereal crop .The cultivated area reached about 3 million feddens in the year of 2011/2012. Wheat is the main human staple food for urban and rural societies and a major source of straw as fodder for animal feeding.

The national wheat production is insufficient to meet our local consumption since no feasible to increase the area devoted to wheat in old lands and thinking to the solution seems to be expanding into new lands which characterized by its low fertility, and due to the shortage of available irrigation water this also a main opstical as well as many other problems.

Increasing production per unit area appears to be the mainly possible alternative of reducing the wheat production gap. The required yield increases may be achieved by introducing high-yielding cultivars and simultaneously implementing improved cultural practices. Such improved varieties must resist or tolerate the unfavorable environments and be stable in broad spectrum of environments.

Since mid-1970's, the Egyptian wheat program has applied the strategy of separating macro-environment differences such as temperature, rainfall and soil type to a closely identified sub-regions with a relatively uniform environment with their recommended cultivars. According to this strategy, five main macroclimatic zones were identified namely, North Delta, South Delta, Middle Egypt, Upper Egypt (i.e. the old land) and Out Valley (the new reclaimed area) at West Delta and New Valley.The first two zones are usually subjected to rust diseases ( stripe ,leaf and stem rusts) while, Upper Egypt is under heat stress. In addition, the main stress prevailing in the new lands may be drought, salinity and heat stresses. Meanwhile, Middle Egypt zone characterized by its high fertility. One or more of these stresses in the different zones are being considered in the National Wheat Research Program aiming to grow more than one cultivar in each region to overcome such stresses.

Current policy of the Egyptian National Wheat Program favors use of more than one cultivar in a given location therefore, more varieties with wider adaptability are thought to improve yield potentially. However, developing narrowly adapted or site specific varieties to specific zones or locations must not be ignored.

The National Wheat Research Program had released the two varieties Giza 157 and Sakha 8 in mid -1970's (Gomma et al., 1979). Giza 157 was recommended for South Delta and Middle Egypt, while Sakha 8 was released for salinity affected soils .

In the early 1980's, two bread wheat varities were released, Sakha 61 for Delta region and Sakha 69 to be planted allover Egypt due to its wider adaptability (Gomma et al., 1984). Moreover, five more new bread wheat cultivars were released during the nineties namely Gemmeiza 1 and Giza 165 (Abdel-Shafi et al., 1991); Sids 1 (Ghanem et al., 1996), Gemmeiza 3 (ElSayed et al., 1997) and Gemmeiza 5 (Mitkees et al., 1998). 
The Egyptian wheat breeders through their continuous efforts had released the two cultivars Sakha 93 and Giza 168 which were characterized by their high resistance to stripe rust and high yielding ability (Shehab El-Din et al., 1999). In addition, the wheat cultivar Gemmeiza 9 proved its superiority as compared with the commercial wheat cultivars Sakha 93, Sids 1 and Gemmeiza 5 as well as its highly resistance to the three rust diseases (Mosaad et al., 2000). In different successive series, further bread wheat cultivars were released, i.e. Gemmeiza 10 (El-Shami et al., 2005), Sakha 94 (Shehab El-Din et al., 2005) , Sids 12 (Mahrous et al., 2009) and Sids 13 (Moustafa et al., 2010).

Following up grain yield of the released cultivars over the past 50 years ensure that the new cultivars always yielded more than the old ones. This genetic yield increase amounted $211 \%$ with $4.2 \%$ increase per year during the 50 years period since 1950 till the year of 2000 (Ageez et al., 1999). Wheat yield have increased gradually over the past 50 years from 4.95 $\mathrm{ard} / \mathrm{fed}$ in the year 1950 to $17.8 \mathrm{ard} / \mathrm{fed}$ in the year 2000 mainly due to the continuous genetic improvement of wheat germplasm in the breeding program.

The objective of this work is to assess grain yield and stability parameters for the new bread wheat cultivar Gemmeiza 11 compared to the superior Egyptian commercial wheat cultivars in five agro-climatic zones of the old lands as well as the new reclaimed areas.

\section{MATERIALS AND METHODS}

The newly bread wheat cultivar Gemmeiza 11 has been selected from a local cross made by wheat breeders at Gemmeiza Agricultural Research Station, Wheat Research Dept. ARC., using the pedigree method. The pedigree and selection history of this cross is:

Bow"s"/ Kvz // 7C / Seri 82 /3/ Giza 168 / Sakha 61

CGM 7892 - 2GM-1GM-2GM-OGM

Gemmeiza 11 was advanced to Gemmeiza wheat screening nursery in 2004/2005 growing season. Afterwards, Gemmeiza 11 was tested in 10 preliminary yield trials in 2005/2006 season in the National Wheat Research Program compared with the commercial wheat cultivars dominated through this period. In 2006/2007 and 2007/2008 growing seasons, Gemmeiza 11 was evaluated in the advanced yield trials where 65 advanced yield trials were conducted at North Delta ,South Delta, Middle Egypt, Upper Egypt and Out Valley .

The experimental plot area was 6 rows, $4 \mathrm{~m}$ long and $20 \mathrm{~cm}$ apart $\left(4.8 \mathrm{~m}^{2}\right)$ in the screening and preliminary yield trials and the harvested area was $\left(3.2 \mathrm{~m}^{2}\right)$ after removing the two outer rows to eliminate the border effect. Meanwhile, the plot area was $10.5 \mathrm{~m}^{2}$ in the advanced yield trials. The Randomized Complete Block Design (RCBD) with 4 replicates was used according to Steel and Torrie (1980) in both preliminary and advanced yield trials. Moreover, stability parameters for grain yield of the advanced yield 
trials were calculated according to Eberhart and Russell (1966).The recommended cultural practices for each region were applied in all experiments.

Also, taking other type for evaluating the new released cultivar on the farmer's levels, 61 verification yield trials including Gemmeiza 11 and 3 dominated wheat cultivars as a checks were carried out in old lands of Delta region and Middle Egypt as well as in the new lands in the three successive seasons 2009/2010, 2010/2011 and 2011/2012 with the cooperation of the Experimental Research Section. The area of each experimental plot was (150 $\mathrm{m}^{2}$ ) for each cultivar planted with the conventional method usually used by the farmers (broadcasting). At harvesting time, four randomly selected samples $4 \mathrm{~m}^{2}$ area from each cultivar were harvested and grain yield was weighted and adjusted to ard /fed.

Taking in consideration the demonstration fields conducted through the co-operation among National Wheat Research Program, Academic Science Research and Technology as well as the Extension Services, where 108 demonstration fields were carried out in the old lands of Delta region in 2011/2012 season including the new released cultivar Gemmeiza 11 along with 5 dominated wheat cultivars. The area of each selected field was at least 1 fed. The demonstration plots were planted in hills $10 \mathrm{~cm}$ apart on raised beds and the width of beds was $120 \mathrm{~cm}$. compared to the conventional method usually used by the farmers (flat planting). At harvesting time, 4 randomly selected samples $\left(4 \mathrm{~m}^{2}\right)$ from each tested cultivar were harvested in and out the demonstration fields and threshed. The grain yield of each sample was weighted and adjusted to ard/fed.

\section{RESULTS AND DISCUSSION}

\section{1- Preliminary yield trials:-}

The results in Table (1-a) showed that the grain yield (ard/fad) of the new bread wheat cultivar Gemmeiza 11 exceeded the yield of the check cultivar Sakha93 at EL-Gemmeiza, Etai EL-Barood and Kafr-EL-Hamam locations as well as the mean of the cultivar Giza 168 over the checks mean in Delta region. Grain yield of the cultivar Gemmeiza 11 exceeded the yield of all check cultivars at Middle Egypt, i.e. Sids and Mallawy (Table 1-b). Meanwhile, at El-Nubaria, the cultivar Gemmeiza 11 out- yielded all check cultivars except the cultivar Gemmeiza 9. At the same time, grain yield of Gemmeiza 11 exceeded the yield of the check cultivars and the checks mean at EL-Matanaa and Kom-Ombo locations in Upper Egypt (Table 1-c). At level of over all Egypt (Table 1-d), the new cultivar proved its superiority over all the check cultivars at Middle and Upper Egypt while, at the new lands in Out Valley, Gemmeiza11 exceeded Sakha 93, Sakha 94 and Giza 168 while, Gemmeiza 9 was the best yielder in Out Valley and may be considered as a drought tolerance variety. 
Table (1-a): Grain yield (ard. /fed.) of the preliminary yield trials for Gemmeiza 11 and four bread wheat cultivars in Delta Region in 2005/2006 season.

\begin{tabular}{|l|c|c|c|c|c|}
\hline \multirow{2}{*}{ Cultivars } & \multicolumn{4}{c|}{ Locations } & \multirow{2}{*}{ Mean } \\
\cline { 2 - 5 } & Sakha & El-Gemmeiza & $\begin{array}{c}\text { Etai } \\
\text { El-Barood }\end{array}$ & $\begin{array}{c}\text { Kafr } \\
\text { El-Hamam }\end{array}$ & \\
\hline Sakha 93 & 23.95 & 19.75 & 22.53 & 18.03 & 21.08 \\
\hline Sakha 94 & 24.85 & 20.17 & 29.75 & 24.35 & 24.78 \\
\hline Giza 168 & 22.88 & 21.74 & 26.58 & 21.96 & 23.29 \\
\hline Gemmeiza 9 & 23.36 & 21.66 & 26.80 & 23.80 & 23.90 \\
\hline Checks mean & 23.76 & 20.83 & 26.42 & 22.04 & 23.26 \\
\hline Gemmeiza 11 & 22.05 & 21.33 & 29.31 & 21.20 & 23.47 \\
\hline L.S.D 5\% & 1.73 & 2.16 & 2.73 & 3.62 & 2.66 \\
\hline C.V \% & 5.52 & 7.85 & 7.73 & 12.08 & 8.56 \\
\hline
\end{tabular}

Table (1-b): Grain yield (ard. /fed.) of the preliminary yield trials for Gemmeiza 11 and four bread wheat cultivars in Middle Egypt and Out Valley in 2005/2006 season.

\begin{tabular}{|l|c|c|c|c|c|}
\hline \multirow{2}{*}{ Cultivars } & \multicolumn{3}{|c|}{ Locations } & Out Valley & \multirow{2}{*}{ Mean } \\
\cline { 2 - 5 } & Sids & Mallawy & $\begin{array}{c}\text { Mean } \\
\text { Middle Egypt }\end{array}$ & El-Nubaria & \\
\hline Sakha 93 & 23.84 & 18.54 & 21.19 & 15.89 & 19.87 \\
\hline Sakha 94 & 22.75 & 21.23 & 21.99 & 17.75 & 20.95 \\
\hline Giza 168 & 23.30 & 19.75 & 21.53 & 17.68 & 20.57 \\
\hline Gemmeiza 9 & 22.86 & 18.97 & 20.91 & 20.96 & 20.93 \\
\hline Checks mean & 23.19 & 19.64 & 21.41 & 18.07 & 20.58 \\
\hline Gemmeiza 11 & $\mathbf{2 5 . 0 9}$ & $\mathbf{2 3 . 3 5}$ & $\mathbf{2 4 . 2 2}$ & $\mathbf{1 8 . 7 7}$ & $\mathbf{2 2 . 8 6}$ \\
\hline L.S.D 5\% & 2.87 & 3.20 & 3.04 & 2.57 & \\
\hline C.V \% & 9.22 & 12.25 & 10.62 & 11.12 & \\
\hline
\end{tabular}

Table (1-c): Grain yield ( ard / fed.) of the preliminary yield trials for Gemmeiza 11 and four bread wheat cultivars in Upper Egypt in 2005/2006 season.

\begin{tabular}{|l|c|c|c|c|}
\hline \multirow{2}{*}{ Cultivars } & \multicolumn{3}{|c|}{ Locations } & \multirow{2}{*}{ Mean } \\
\cline { 2 - 4 } & Shandaweel 1 & El-Matanaa & Kom- Ombo & \\
\hline Sakha 93 & 18.46 & 17.44 & 22.27 & 19.39 \\
\hline Sakha 94 & 23.61 & 16.05 & 23.92 & 21.20 \\
\hline Giza 168 & 22.37 & 18.68 & 22.67 & 21.24 \\
\hline Gemmeiza 9 & 20.85 & 14.85 & 20.76 & 18.82 \\
\hline Checks mean & 21.32 & 16.76 & 22.41 & 20.16 \\
\hline Gemmeiza 11 & 21.65 & 19.08 & 23.94 & 21.55 \\
\hline L.S.D 5\% & 2.53 & 2.77 & 3.62 & 4.19 \\
\hline C.V \% & 8.75 & 25.70 & 13.34 & 16.03 \\
\hline
\end{tabular}


Table (1-d): Grain yield (ard / fed.) of the preliminary yield trials for Gemmeiza 11 and four bread wheat cultivars in over all Egypt in 2005/2006 season.

\begin{tabular}{|l|c|c|c|c|c|}
\hline \multirow{2}{*}{ Cultivars } & \multicolumn{4}{|c|}{ Locations } & \multirow{2}{*}{ Mean } \\
\cline { 2 - 5 } & Delta & Middle Egypt & $\begin{array}{c}\text { Upper } \\
\text { Egypt }\end{array}$ & Out Valley & 19.39 \\
\hline Sakha 93 & 21.08 & 21.19 & 19.39 & 15.89 & 21.43 \\
\hline Sakha 94 & 24.78 & 21.99 & 21.20 & 17.75 & 20.94 \\
\hline Giza 168 & 23.29 & 21.53 & 21.24 & 17.68 & 21.15 \\
\hline Gemmeiza 9 & 23.90 & 20.91 & 18.82 & 20.96 & 20.73 \\
\hline Checks mean & 23.26 & 21.41 & 20.16 & 18.07 & $\mathbf{2 2 . 0 0}$ \\
\hline Gemmeiza 11 & $\mathbf{2 3 . 4 7}$ & $\mathbf{2 4 . 2 2}$ & $\mathbf{2 1 . 5 5}$ & $\mathbf{1 8 . 7 7}$ & 0.95 \\
\hline L.S.D 5\% & 2.66 & 3.04 & 4.19 & 2.57 & 9.83 \\
\hline C.V \% & 8.56 & 10.62 & 16.03 & 11.12 & \\
\hline
\end{tabular}

\section{2-Adanced yield trials:}

The average grain yield of Gemmeiza 11 compared to the commercial cultivars, Sakha 93 , Sakha 94 , Gemmeiza 9 and Giza 168 in 65 advanced yield trials conducted at different five main macroclimatic zones in 2006/2007 and 2007/2008 growing seasons are shown in Tables 2 and 3 ,respectively . The yield of Gemmeiza 11 surpassed the yield of checks mean of the cultivars Gemmeiza 9 and Giza 168 and the cultivar means over 6 locations means at North Delta in 2006 / 2007 season (Table 2-a). The average increase of Gemmeiza 11 in North Delta was 5.5\% above the mean of all check cultivars.

Data in Table (2-b) showed that the newly bread wheat cultivar had significantly exceeded the checks mean at South Delta in all locations with an average increase of $11 \%$ and exceeded all check cultivars at EL-Gemmeiza, Sharqia 2, Monufia and Qalyobia governorates. Meanwhile, grain yield of the newly released cultivar yielded almost the same as the checks mean cultivars at Middle Egypt (Table 2-c) while, surpassed the yield of the check means at the locations of Upper Egypt especially at Kom- Ombo (Table 2-d).

The data presented in Table (2-e) showed the superiority of the new released cultivar Gemmeiza 11 at Sakha, Gemmeiza and Sids locations in the advanced yield trials in the case of drilling sowing with an increase of 9.2 $\%$ when compared with the mean of all tested locations. The Out Valley results of the advanced yield trials in 2006/2007 season illustrated in Table (2-f) revealed that Gemmeiza11 had significantly exceeded all the check cultivars only at EL-Ismailia location and Gemmieza 9 at Asyut and Toshkey. Meanwhile, Gemmeiza 11 grain yield was not significantly differed from the commercial checks at EL-Nubaria and EL-Eoinaat locations .The average increase of the new cultivar means over all locations at Out Valley was only $1 \%$. The results of the advanced yield trials over all Egypt in 2006/2007 season cleared that the new cultivar had significantly out-yielded the check cultivars Gemmeiza 9 and Giza 168 at North and South Delta and Upper Egypt as well as Sakha 93 and Sakha 94 at South Delta and Gemmeiza 9 in Out Valley. The average increase in grain yield of Gemmeiza 11 above all the means of the check cultivars at over all Egypt was $5.9 \%$ ( Table 2-j). 
Table (2-a): Grain yield (ard / fed.) of the advanced yield trials ( D-BW)for Gemmeiza 11 and four bread wheat cultivars in North Delta in $2006 / 2007$ season.

\begin{tabular}{|c|c|c|c|c|c|c|c|c|}
\hline \multirow[b]{2}{*}{ Cultivars } & \multicolumn{7}{|c|}{ Locations } & \multirow[b]{2}{*}{ Mean } \\
\hline & $\begin{array}{l}\text { El- } \\
\text { Serw }\end{array}$ & Sakha & \begin{tabular}{|c|} 
Tag \\
El-Ezz
\end{tabular} & $\begin{array}{c}\text { Dakahlia } \\
-1\end{array}$ & $\begin{array}{c}\text { Dakahlia } \\
-2\end{array}$ & $\begin{array}{c}\text { Etai } \\
\text { El- } \\
\text { Barood }\end{array}$ & Beheira & \\
\hline Sakha 93 & 13.72 & 26.66 & 22.67 & 17.67 & 20.19 & 21.04 & 24.64 & 20.94 \\
\hline Sakha 94 & 16.36 & 27.05 & 21.40 & 17.00 & 20.36 & 24.87 & 21.97 & 21.29 \\
\hline Gemmeiza 9 & 13.34 & 25.58 & 19.40 & 12.00 & 20.96 & 24.54 & 18.57 & 19.20 \\
\hline Giza 168 & 15.76 & 27.43 & 19.27 & 14.67 & 17.35 & 23.90 & 23.67 & 20.29 \\
\hline Checks mean & 14.80 & 26.68 & 20.69 & 15.34 & 19.72 & 23.59 & 22.21 & 20.43 \\
\hline Gemmeiza 11 & 17.53 & 28.00 & 19.67 & 15.67 & 22.61 & 24.00 & 23.34 & 21.55 \\
\hline L.S.D 5\% & 3.85 & 1.86 & 3.52 & 2.63 & 3.39 & 2.31 & 0.99 & 1.04 \\
\hline C.V \% & 16.74 & 4.7 & 12.35 & 12.66 & 12.24 & 6.68 & 3.11 & 9.56 \\
\hline
\end{tabular}

Table (2-b): Grain yield (ard / fed.) of the advanced yield trials (D-BW) for Gemmeiza 11 and four bread wheat cultivars in South Delta in 2006/2007 season.

\begin{tabular}{|l|c|c|c|c|c|c|c|c|}
\hline \multirow{3}{*}{ Cultivars } & \multicolumn{7}{|c|}{ Locations } & \\
\cline { 2 - 10 } & Gemmeiza & $\begin{array}{c}\text { Sers } \\
\text { El- } \\
\text { Liaan }\end{array}$ & $\begin{array}{c}\text { Kafr } \\
\text { El- } \\
\text { Hamam }\end{array}$ & $\begin{array}{c}\text { Sharkia } \\
-\mathbf{1}\end{array}$ & $\begin{array}{c}\text { Sharkia } \\
-\mathbf{2}\end{array}$ & Monufia & Qaluobia & Mean \\
\hline Sakha 93 & 25.50 & 26.80 & 22.80 & 16.85 & 21.03 & 21.60 & 23.87 & 22.64 \\
\hline Sakha 94 & 27.04 & 21.47 & 22.01 & 19.92 & 24.17 & 26.07 & 23.17 & 23.41 \\
\hline Gemmeiza9 & 24.94 & 20.04 & 25.48 & 19.86 & 18.46 & 26.60 & 28.14 & 23.36 \\
\hline Giza 168 & 27.30 & 22.84 & 21.81 & 17.70 & 23.65 & 27.94 & 26.40 & 23.95 \\
\hline Checks mean & 26.20 & 22.79 & 23.03 & 18.58 & 21.83 & 25.55 & 25.40 & 23.34 \\
\hline Gemmeiza 11 & 27.57 & 26.37 & 24.16 & 18.43 & 26.91 & 28.60 & 29.40 & 25.92 \\
\hline L.S.D 5\% & - & 3.11 & 2.71 & 1.96 & 2.64 & 4.07 & - & 1.22 \\
\hline C.V\% & 5.07 & 8.79 & 8.16 & 7.47 & 8.09 & 11.06 & 14.60 & 9.67 \\
\hline
\end{tabular}

Table (2-c):Grain yield (ard / fed.) of the advanced yield trials (D-BW) for Gemmeiza 11 and four bread wheat cultivars in Middle Egypt in 2006/ 2007 season.

\begin{tabular}{|l|c|c|c|c|c|c|}
\hline \multirow{2}{*}{ Cultivars } & \multicolumn{5}{|c|}{ Locations } & \multirow{2}{*}{ Mean } \\
\cline { 2 - 6 } & Giza & Fayoum & Sids & Mallawy & Menia & \\
\hline Sakha 93 & 26.74 & 26.34 & 30.20 & 21.88 & 23.10 & 25.65 \\
\hline Sakha 94 & 29.75 & 27.00 & 28.67 & 19.50 & 21.35 & 25.26 \\
\hline Gemmeiza 9 & 26.88 & 27.34 & 28.57 & 19.66 & 23.10 & 25.11 \\
\hline Giza 168 & 29.19 & 28.47 & 28.64 & 19.58 & 24.50 & 26.08 \\
\hline Checks mean & 28.14 & 27.29 & 29.02 & 20.16 & 23.01 & 25.52 \\
\hline Gemmeiza 11 & 23.45 & 30.47 & 29.87 & 20.97 & 21.00 & 25.15 \\
\hline L.S.D 5\% & 2.27 & - & - & 2.69 & 2.91 & 1.13 \\
\hline C.V\% & 6.01 & 7.82 & 4.76 & 9.15 & 9.53 & 7.31 \\
\hline
\end{tabular}


Table (2-d):Grain yield (ard / fed.) of the advanced yield trials (D-BW) for Gemmeiza 11 and four bread wheat cultivars in Upper Egypt in 2006/2007 season.

\begin{tabular}{|l|c|c|c|c|}
\hline \multirow{2}{*}{ Cultivars } & \multicolumn{3}{c|}{ Locations } & \multirow{2}{*}{ Mean } \\
\cline { 2 - 4 } & Shandaweel 1 & El-Matanaa & Kom-Ombo & 19.65 \\
\hline Sakha 93 & 20.84 & 23.47 & 14.65 & 22.01 \\
\hline Sakha 94 & 22.70 & 23.60 & 19.71 & 19.34 \\
\hline Gemmeiza 9 & 17.71 & 23.27 & 17.05 & 20.20 \\
\hline Giza 168 & 19.17 & 24.27 & 17.16 & 20.3 \\
\hline Checks mean & 20.11 & 23.65 & 17.14 & 23.02 \\
\hline Gemmeiza 11 & 20.87 & 24.74 & 23.44 & 1.62 \\
\hline L.S.D 5\% & 2.02 & - & 3.24 & 9.37 \\
\hline C.V\% & 6.89 & 9.03 & 11.86 & \\
\hline
\end{tabular}

Table (2- e): Grain yield (ard / fed.) of the advanced yield trials (D-BW)Drill for Gemmeiza11 and four bread wheat cultivars in 2006/2007 season.

\begin{tabular}{|l|c|c|c|c|c|c|}
\hline \multirow{2}{*}{ Cultivar } & \multicolumn{5}{|c|}{ Locations } & \multirow{2}{*}{ Mean } \\
\cline { 2 - 6 } & Sakha & Gemmeiza & Bahteem & Sids & Shandaweel & \\
\hline Sakha 93 & 28.38 & 28.34 & 12.14 & 31.37 & 24.86 & 25.02 \\
\hline Sakha 94 & 28.69 & 28.34 & 12.60 & 28.87 & 32.88 & 26.28 \\
\hline Gemmeiza 9 & 26.47 & 24.47 & 13.54 & 29.34 & 28.18 & 24.40 \\
\hline Giza 168 & 29.68 & 27.64 & 14.04 & 27.37 & 28.32 & 25.42 \\
\hline Checks mean & 28.31 & 27.20 & 13.08 & 29.24 & 28.56 & 25.28 \\
\hline Gemmeiza 11 & 30.47 & 30.70 & 13.67 & 31.27 & 31.95 & 27.61 \\
\hline L.S.D 5\% & 1.68 & 1.96 & 1.24 & 2.32 & 2.88 & 0.91 \\
\hline C.V\% & 4.07 & 4.77 & 6.67 & 5.29 & 6.47 & 5.58 \\
\hline
\end{tabular}

Table (2-f): Grain yield (ard / fed.) of the advanced yield trials (D-BW) for Gemmeiza 11 and four bread wheat cultivars in out Vally in 2006/2007 season.

\begin{tabular}{|c|c|c|c|c|c|c|c|}
\hline \multirow[b]{2}{*}{ Cultivar } & \multicolumn{6}{|c|}{ Locations } & \multirow[b]{2}{*}{ Mean } \\
\hline & Nubaria & Ismailia & Assuit & $\begin{array}{c}\text { New } \\
\text { Valley }\end{array}$ & Toshkey & Eoinaat & \\
\hline Sakha 93 & 26.65 & 7.27 & 14.87 & 14.67 & 17.32 & 15.07 & 15.97 \\
\hline Sakha 94 & 27.84 & 9.28 & 14.54 & 17.24 & 14.17 & 14.87 & 16.32 \\
\hline Gemmeiza 9 & 26.50 & 7.76 & 11.67 & 16.04 & 12.69 & 11.44 & 14.35 \\
\hline Giza 168 & 26.46 & 10.18 & 14.47 & 14.80 & 15.96 & 13.30 & 15.86 \\
\hline Checks mean & 26.86 & 8.62 & 13.89 & 15.69 & 15.04 & 13.67 & 15.63 \\
\hline Gemmeiza 11 & 25.19 & 11.34 & 15.07 & 14.70 & 15.66 & 12.77 & 15.79 \\
\hline L.S.D 5\% & - & 0.55 & 1.79 & 1.79 & 2.55 & - & 0.90 \\
\hline C.V \% & 8.16 & 3.79 & 8.64 & 8.00 & 12.14 & 4.19 & 9.95 \\
\hline
\end{tabular}

Table (2-j): Grain yield (ard / fed.) of the advanced yield trials (D-BW) for Gemmeiza 11 and four bread wheat cultivars in over all Egypt in 2006/2007 season

\begin{tabular}{|c|c|c|c|c|c|c|c|}
\hline \multirow{2}{*}{ Cultivar } & \multicolumn{6}{|c|}{ Locations } & \multirow{2}{*}{ Mean } \\
\hline & N. Delta & S. Delta & M. Egypt & U. Egypt & Out Valley & Drill & \\
\hline Sakha 93 & 20.94 & 22.64 & 25.65 & 19.65 & 15.97 & 25.02 & 21.65 \\
\hline Sakha 94 & 21.29 & 23.41 & 25.26 & 22.01 & 16.32 & 26.28 & 22.43 \\
\hline Gemmeiza 9 & 19.20 & 23.36 & 25.11 & 19.34 & 14.35 & 24.40 & 20.96 \\
\hline Giza 168 & 20.29 & 23.95 & 26.08 & 20.20 & 15.86 & 25.42 & 21.97 \\
\hline Checks mean & 20.43 & 23.34 & 25.53 & 20.30 & 15.63 & 25.28 & 21.75 \\
\hline Gemmeiza 11 & 21.55 & 25.92 & 25.15 & 23.02 & 15.79 & 27.61 & 23.03 \\
\hline L.S.D 5\% & 1.04 & 1.22 & 1.13 & 1.62 & 0.90 & 0.91 & 0.46 \\
\hline C.V \% & 9.56 & 9.67 & 7.31 & 9.37 & 9.95 & 5.58 & 8.61 \\
\hline
\end{tabular}


The advanced yield trials carried out at North Delta in 2007/2008 growing season (Table 3-a), showed that the mean grain yield of the new wheat cultivar Gemmeiza 11 was 20.86 ard. /fed. ensuring equal potentiality with the dominating wheat cultivars i.e. Sakha 93, Gemmeiza 9 and Giza 168 at North Delta region. In addition, the yield of the new cultivar surpassed significantly the mean yield of the check cultivars at South Delta region with a value of $8.9 \%$ (Table $3-b$ ).

Furthermore, the new cultivar scored the highest value for yield at ElFaiyum, Sids and Mallawy locations being 34.13, 27.40 and 26.06 ard/fad., respectively (Table 3-c) with a mean yield increment of $11.2 \%$ over all the checks cultivar means at Middle Egypt region. Regarding Upper Egypt (Table 3-d),the new cultivar Gemmeiza 11 yielded the same as the check cultivars at Shandaweel location while, surpassed all of them at El-Matanaa and exceeded only Gemmeiza 9 at Kom -Ombo location. The increase in mean grain yield of the new cultivar reached about $6.2 \%$ over the checks mean at Upper Egypt. In respect to the new lands( Table 3-f), Gemmeiza 11 grain yield significantly surpassed Gemmeiza 9 in 4 locations, Sakha 93 and Giza 168 in 3 locations and Shandaweel 1 at Nubaria and New Valley locations. The mean yield increment of the new cultivar at Out Valley was $20.3 \%$ above the mean of all the check cultivars.

The grain yield (ard. /fed.) of the advanced yield trials sown using drilling method for Gemmeiza 11 and four bread wheat cultivars in 2007/2008 growing season are presented in Table (3-e). The new cultivar proved its superiority above the check cultivars Sakha 93, Shandaweel 1, Gemmeiza 9 and Giza 168 at Sakha, Gemmeiza and Sids locations. The average yield increament of Gemmeiza 11 was $1.42 \mathrm{ard} / \mathrm{fed}$. (6.05\%) when compared with the means of all check cultivars across all tested locations. The new cultivar out-yielded the mean of all the check cultivars in 32 advanced yield trials at over all Egypt in 2007/2008 season by $9.6 \%$ (Table 3 - j). These results indicate that the maximum grain yield potentiality is expected to be obtained from the new cultivar Gemmeiza 11 when will be grown in South Delta, Middle Egypt and Out Valley.

Table (3-a): Grain yield (ard / fed.) of the advanced yield trials (D-BW) for Gemmeiza11 and four bread wheat cultivars in North Delta in 2007/2008 season.

\begin{tabular}{|c|c|c|c|c|c|c|c|}
\hline \multirow[b]{2}{*}{ Cultivars } & \multicolumn{6}{|c|}{ Locations } & \multirow[b]{2}{*}{ Mean } \\
\hline & El-Serw & $\begin{array}{c}\text { Dakahlia } \\
-1\end{array}$ & $\begin{array}{c}\text { Dakahlia } \\
-2\end{array}$ & Sakha & \begin{tabular}{|c|} 
Etai \\
El-Barood
\end{tabular} & Beheira & \\
\hline Sakha 93 & 14.45 & 16.87 & 19.07 & 23.29 & 23.47 & 26.60 & 20.63 \\
\hline Shandaweel 1 & 13.39 & 19.47 & 22.67 & 20.30 & 24.54 & 29.00 & 21.56 \\
\hline Gemmeiza 9 & 13.34 & 17.60 & 20.34 & 20.84 & 24.74 & 21.34 & 19.70 \\
\hline Giza 168 & 12.96 & 17.27 & 21.00 & 21.01 & 22.80 & 25.07 & 20.02 \\
\hline Checks mean & 13.54 & 17.80 & 20.77 & 21.36 & 23.89 & 25.50 & 20.48 \\
\hline Gemmeiza 11 & 14.21 & 18.94 & 23.07 & 22.73 & 23.84 & 22.34 & 20.86 \\
\hline L.S.D 5\% & 1.80 & 1.56 & 1.35 & 2.18 & 1.59 & 1.95 & 0.70 \\
\hline C.V \% & 9.51 & 6.32 & 4.74 & 6.99 & 4.62 & 5.57 & 6.11 \\
\hline
\end{tabular}


Table (3-b): Grain yield (ard / fed.) of the advanced yield trials (D-BW) for Gemmeiza 11 and four bread wheat cultivars in South Delta in 2007/2008 season.

\begin{tabular}{|l|c|c|c|c|c|c|c|c|c|}
\hline \multirow{2}{*}{ Cultivars } & \multicolumn{7}{|c|}{ Locations } & \\
\cline { 2 - 11 } & Gemmeiza & $\begin{array}{c}\text { Sers } \\
\text { El- } \\
\text { Liaan }\end{array}$ & $\begin{array}{c}\text { Tag } \\
\text { El- } \\
\text { Ezz }\end{array}$ & $\begin{array}{c}\text { Kafr-EI- } \\
\text { Hamam }\end{array}$ & $\begin{array}{c}\text { Sharkia } \\
-\mathbf{1}\end{array}$ & $\begin{array}{c}\text { Sharkia } \\
-\mathbf{2}\end{array}$ & Monufia & Qaluobia & Mean \\
\hline Sakha 93 & 24.58 & 26.17 & 16.90 & 20.05 & 21.64 & 16.54 & 23.60 & 28.67 & 22.27 \\
\hline Shandaweel 1 & 24.73 & 28.70 & 21.76 & 16.47 & 18.20 & 22.07 & 22.60 & 33.07 & 23.45 \\
\hline Gemmeiza 9 & 24.26 & 21.94 & 19.06 & 18.98 & 20.17 & 19.27 & 24.80 & 26.54 & 21.88 \\
\hline Giza 168 & 24.29 & 26.37 & 20.10 & 20.65 & 21.40 & 19.14 & 24.20 & 27.94 & 23.01 \\
\hline Checks mean & 24.47 & 25.80 & 19.46 & 19.04 & 20.35 & 19.26 & 23.80 & 29.06 & 22.65 \\
\hline Gemmeiza 11 & 26.78 & 29.60 & 22.71 & 21.07 & 21.20 & 18.54 & 28.14 & 29.34 & 24.67 \\
\hline L.S.D 5\% & 1.45 & 4.04 & 1.52 & 1.82 & n.S & 2.53 & n.s & n.s & 1.06 \\
\hline C.V \% & 4.03 & 10.49 & 5.73 & 6.92 & 8.11 & 8.51 & 14.41 & 9.73 & 9.51 \\
\hline
\end{tabular}

Table (3-c): Grain yield (ard / fed.) of the advanced yield trials (D-BW) for Gemmeiza 11 and four bread wheat cultivars in Middle Egypt in 2007/2008 season.

\begin{tabular}{|l|c|c|c|c|c|c|}
\hline \multirow{2}{*}{ Cultivars } & \multicolumn{5}{|c|}{ Locations } & \multirow{2}{*}{ Mean } \\
\cline { 2 - 6 } & Giza & Fayoum & Sids & Mallawy & Menia & \\
\hline Sakha 93 & 25.00 & 31.85 & 26.07 & 20.58 & 20.33 & 24.77 \\
\hline Shandaweel 1 & 25.67 & 32.38 & 26.00 & 20.32 & 15.41 & 23.96 \\
\hline Gemmeiza 9 & 25.00 & 33.95 & 22.40 & 19.78 & 19.62 & 24.15 \\
\hline Giza 168 & 26.34 & 30.80 & 22.14 & 21.60 & 22.76 & 24.73 \\
\hline Checks mean & 25.50 & 32.25 & 24.15 & 20.57 & 19.53 & 24.40 \\
\hline Gemmeiza 11 & 25.34 & 34.13 & 27.40 & 26.06 & 22.75 & 27.13 \\
\hline L.S.D 5\% & 2.07 & 4.40 & 1.43 & 2.37 & 1.30 & 1.12 \\
\hline C.V\% & 5.61 & 9.50 & 3.87 & 7.22 & 4.39 & 7.03 \\
\hline
\end{tabular}

Table (3-d): Grain yield (ard / fed.) of the advanced yield trials (D-BW) for Gemmeiza 11 and four bread wheat cultivars in Upper Egypt in 2007/2008 season.

\begin{tabular}{|l|c|c|c|c|}
\hline \multirow{2}{*}{ Cultivars } & \multicolumn{3}{|c|}{ Locations } & \multirow{2}{*}{ Mean } \\
\cline { 2 - 4 } & Shandaweel 1 & El-Matanaa & Kom- Ombo & 20.19 \\
\hline Sakha 93 & 17.19 & 22.74 & 20.66 & 22.35 \\
\hline Shandaweel 1 & 18.57 & 26.74 & 21.75 & 17.76 \\
\hline Gemmeiza 9 & 17.50 & 19.94 & 15.83 & 19.97 \\
\hline Giza 168 & 17.57 & 23.67 & 18.68 & 20.07 \\
\hline Checks mean & 17.71 & 23.27 & 19.23 & 21.32 \\
\hline Gemmeiza 11 & 17.87 & 27.67 & 18.41 & 1.91 \\
\hline L.S.D 5\% & 2.73 & 3.81 & 3.56 & 11.55 \\
\hline C.V\% & 10.16 & 11.00 & 13.30 & \\
\hline
\end{tabular}

Table (3-e): Grain yield (ard / fed.) of the advanced yield trials (D-BW) Drill for Gemmeiza 11 and four bread wheat cultivars in 2007/2008 season.

\begin{tabular}{|l|c|c|c|c|c|c|}
\hline \multirow{2}{*}{\multicolumn{1}{c|}{ Cultivar }} & \multicolumn{7}{c|}{ Locations } \\
\cline { 2 - 7 } & Sakha & Gemmeiza & Giza & Sids & Shandaweel 1 & Mean \\
\hline Sakha 93 & 23.96 & 27.01 & 18.25 & 30.97 & 18.13 & 23.66 \\
\hline Shandaweel 1 & 23.54 & 27.36 & 17.69 & 29.87 & 22.50 & 24.19 \\
\hline Gemmeiza 9 & 21.37 & 27.44 & 23.94 & 25.40 & 18.17 & 23.26 \\
\hline Giza 168 & 23.32 & 28.68 & 16.53 & 27.47 & 18.26 & 22.85 \\
\hline Checks mean & 23.05 & 27.62 & 19.10 & 28.43 & 19.27 & 23.49 \\
\hline Gemmeiza 11 & 24.96 & 29.16 & 17.00 & 32.40 & 21.02 & 24.91 \\
\hline L.S.D 5\% & 2.31 & n.S & 2.79 & 2.18 & 2.46 & 1.04 \\
\hline C.V \% & 6.71 & 5.40 & 9.96 & 5.08 & 8.45 & 6.85 \\
\hline
\end{tabular}


Table (3-f): Grain yield (ard / fed.) of the advanced yield trials (D-BW) for Gemmeiza 11 and four bread wheat cultivars in Out Valley in 2007/2008 season.

\begin{tabular}{|l|c|c|c|c|c|c|}
\hline \multirow{2}{*}{ Cultivar } & \multicolumn{6}{c|}{ Locations } \\
\cline { 2 - 7 } & Ismailia & Nubaria & Assuit & New Valley & $\begin{array}{c}\text { Abo } \\
\text { Semble }\end{array}$ & Mean \\
\hline Sakha 93 & 10.98 & 21.67 & 11.44 & 20.00 & 7.30 & 14.28 \\
\hline Shandaweel 1 & 17.84 & 18.27 & 13.47 & 18.67 & 7.24 & 15.10 \\
\hline Gemmeiza 9 & 9.94 & 17.87 & 9.40 & 18.20 & 6.57 & 12.40 \\
\hline Giza 168 & 19.14 & 23.67 & 12.40 & 13.94 & 8.25 & 15.48 \\
\hline Checks mean & 14.48 & 20.37 & 11.68 & 17.70 & 7.34 & 14.31 \\
\hline Gemmeiza 11 & 18.12 & 26.27 & 14.60 & 20.10 & 6.99 & 17.22 \\
\hline L.S.D 5\% & 1.02 & 2.71 & 2.06 & 1.78 & 0.95 & 0.79 \\
\hline C.V \% & 4.29 & 8.70 & 10.57 & 6.62 & 9.45 & 8.20 \\
\hline
\end{tabular}

Table (3-j): Grain yield (ard / fed.) of the advanced yield trials (D-BW) for Gemmeiza11 and four bread wheat cultivars in over all Egypt in 2007/2008 season.

\begin{tabular}{|c|c|c|c|c|c|c|c|}
\hline \multirow[b]{2}{*}{ Cultivar } & \multicolumn{6}{|c|}{ Locations } & \multirow[b]{2}{*}{ Mean } \\
\hline & $\begin{array}{l}\text { North } \\
\text { Delta }\end{array}$ & $\begin{array}{l}\text { South } \\
\text { Delta }\end{array}$ & $\begin{array}{c}\text { Middle } \\
\text { Egypt }\end{array}$ & $\begin{array}{l}\text { Upper } \\
\text { Egypt }\end{array}$ & $\begin{array}{c}\text { Out } \\
\text { Valley }\end{array}$ & Drill & \\
\hline Sakha 93 & 20.63 & 22.27 & 24.77 & 20.19 & 14.28 & 23.66 & 20.97 \\
\hline Shandaweel 1 & 21.65 & 23.45 & 23.96 & 22.35 & 15.10 & 24.19 & 21.78 \\
\hline Gemmeiza 9 & 19.70 & 21.88 & 24.15 & 17.76 & 12.40 & 23.26 & 19.86 \\
\hline Giza 168 & 20.02 & 23.01 & 24.73 & 19.97 & 15.48 & 22.85 & 21.01 \\
\hline Checks mean & 20.50 & 22.65 & 24.40 & 20.07 & 14.32 & 23.49 & 20.90 \\
\hline Gemmeiza 11 & 20.86 & 24.67 & 27.13 & 21.32 & 17.22 & 24.91 & 22.90 \\
\hline L.S.D $5 \%$ & 0.7 & 1.06 & 1.12 & 1.91 & 0.79 & 1.04 & 0.43 \\
\hline C.V \% & 6.11 & 9.51 & 7.03 & 11.55 & 8.20 & 6.85 & 8.24 \\
\hline
\end{tabular}

\section{3-Verification yield trials}

Results in Table (4) show the average grain yield estimated for 66 On- Farm yield trials for Gemmeiza 11 and some other commercial cultivars which were carried out at 16 Governorates representing the old and new lands in 2009/2010, 11 Governorates in 2010/2011 and 13 Governorates in 2011/2012.

The results proved that the new wheat cultivar Gemmeiza 11 surpassed the commercial cultivars at the Governorates representing North, Middle and South Delta and the new lands in 2009/2010 growing season. Meanwhile, the yield potentiality of the new cultivar was almost the same as the local checks at Upper Egypt and was less than Sakha 93 and Giza168 at Middle Egypt (Table 4-a).The grain yield of the new released cultivar exceeded the check wheat cultivars at Middle Delta and was equal at South Delta in 2010/2011 growing season (Table 4-b).Meanwhile, in 2011/2012 season, the yield of Gemmeiza 11 surpassed Giza 168 at all tested zones (Table 4-c). On the other hand, the grand mean yield of the new released cultivar Gemmeiza 11 exceeded the grand mean yield of Sakha 93 and Giza 168 by $8.2 \%$ and $3.8 \%$ respectively, through the three successive growing seasons, at over all Delta regions, Middle and Upper Egypt and New Lands while, yielded the same as the check cultivar Gemmeiza 9 (Table 4-d). 


\section{Sadek, Eman M. et al.}

The results cleared the high yielding abilities of the new bread wheat cultivar Gemmeiza 11 and its high yielding adaptability at North, Middle and South Delta as well as at the new lands. Meanwhile, the grain yield of Gemmeiza 11 was less than the commercial wheat cultivars Sakha 93 and Giza 168 at Middle Egypt and was equal at Upper Egypt.

Table (4-a): Grain yield (ard / fed.) of verification yield trials of the newly released cultivar Gemmeiza 11 in 2009 /2010 season.

\begin{tabular}{|c|c|c|c|c|c|c|}
\hline \multirow[b]{2}{*}{ Zones } & \multirow[b]{2}{*}{ Governorates } & \multirow[b]{2}{*}{$\begin{array}{l}\text { No. of } \\
\text { trials }\end{array}$} & \multirow[b]{2}{*}{ Gemmeiza11 } & \multicolumn{3}{|c|}{ Checks } \\
\hline & & & & Sakha 93 & $\begin{array}{c}\text { Giza } \\
168\end{array}$ & Gemmeiza 9 \\
\hline \multirow{3}{*}{ North Delta } & Damietta & 1 & 20.76 & 21.70 & 21.00 & 20.03 \\
\hline & Kafr El -Sheikh & 1 & 23.17 & 18.22 & 21.84 & 19.74 \\
\hline & Alexandria & 1 & 18.69 & 20.90 & 19.47 & 20.90 \\
\hline Mean & & - & 20.87 & 20.27 & 20.77 & 20.22 \\
\hline \multirow{3}{*}{ Middle Delta } & Sharkia & 2 & 19.95 & 16.40 & 17.24 & 14.98 \\
\hline & Dakahlia & 2 & 12.59 & 13.34 & 12.39 & 13.05 \\
\hline & Gharbia & 2 & 21.70 & 21.00 & 20.30 & 18.2 \\
\hline Mean & & - & 18.08 & 16.91 & 16.64 & 15.41 \\
\hline \multirow{3}{*}{ South Delta } & Monufia & 1 & 27.44 & 26.46 & 25.20 & 25.76 \\
\hline & Qalyubia & 2 & 26.87 & 23.88 & 21.11 & 23.27 \\
\hline & Giza & 1 & 23.24 & 23.80 & 29.40 & 24.64 \\
\hline Mean & & - & 25.85 & 24.71 & 25.24 & 24.56 \\
\hline \multirow{2}{*}{ Middle Egypt } & Faiyum & 2 & 17.85 & 22.02 & 21.51 & - \\
\hline & Beni-Suef & 2 & 18.90 & 19.25 & 19.95 & - \\
\hline Mean & & - & 18.38 & 20.64 & 20.73 & \\
\hline \multirow{3}{*}{ Upper Egypt } & Assuit & 2 & 18.48 & 18.46 & 18.20 & - \\
\hline & Sohag & 1 & 17.64 & 18.48 & 21.56 & - \\
\hline & Aswan & 1 & 18.40 & 18.30 & 16.94 & - \\
\hline Mean & & - & 18.17 & 18.41 & 18.90 & \\
\hline \multirow{2}{*}{ New Land } & Port Said & 1 & 15.50 & 09.02 & 10.12 & 13.20 \\
\hline & Suez & 1 & 18.20 & 18.20 & 15.96 & 18.48 \\
\hline Mean & & - & 16.85 & 13.61 & 13.04 & 15.84 \\
\hline \multicolumn{2}{|l|}{ Zones mean } & 23 & 19.70 & 19.09 & 19.22 & 19.01 \\
\hline
\end{tabular}

Table (4-b): Grain yield (ard / fed.) of verification yield trials of the newly released cultivar Gemmeiza 11 in 2010 /2011 season.

\begin{tabular}{|c|c|c|c|c|c|c|}
\hline \multirow{2}{*}{ Zones } & \multirow{2}{*}{ Governorates } & \multirow{2}{*}{$\begin{array}{l}\text { No. of } \\
\text { trials }\end{array}$} & \multirow{2}{*}{ Gemmeiza11 } & \multicolumn{3}{|c|}{ Checks } \\
\hline & & & & Sakha93 & Giza 168 & Gemmeiza9 \\
\hline \multirow{3}{*}{ North Delta } & Damietta & 1 & 12.41 & 8.79 & 13.56 & 14.00 \\
\hline & Kafr EI -Sheikh & 2 & 19.34 & 17.80 & 19.28 & 18.20 \\
\hline & Beheira & 2 & 21.84 & 20.02 & 21.20 & 19.95 \\
\hline Mean & & - & 17.86 & 15.54 & 18.01 & 17.38 \\
\hline \multirow{3}{*}{ Middle Delta } & Sharkia & 2 & 17.10 & 16.05 & 17.76 & 14.0 \\
\hline & Dakahlia & 2 & 20.04 & 18.31 & 14.20 & 20.98 \\
\hline & Gharbia & 2 & 28.00 & 22.80 & 23.40 & 22.40 \\
\hline Mean & & - & 21.71 & 19.05 & 18.45 & 19.13 \\
\hline \multirow{3}{*}{ South Delta } & Monufia & 2 & 22.50 & 23.20 & 19.76 & 23.19 \\
\hline & Qalyubia & 2 & 27.88 & 24.11 & 26.57 & 24.91 \\
\hline & Giza & 2 & 22.10 & 25.20 & 25.90 & 25.90 \\
\hline Mean & & - & 24.16 & 24.17 & 24.08 & 24.67 \\
\hline \multirow{2}{*}{ New Land } & Port Said & 1 & 18.14 & 18.84 & 17.81 & 24.35 \\
\hline & El-Bostan & 2 & 17.96 & 12.07 & 13.67 & 16.21 \\
\hline Mean & & - & 18.05 & 15.46 & 15.74 & 20.28 \\
\hline \multicolumn{2}{|l|}{ Zones mean } & 20 & 20.45 & 18.56 & 19.07 & 20.37 \\
\hline
\end{tabular}


Table (4-c): Grain yield (ard / fed) of verification yield trials of the newly released cultivar Gemmeiza 11 in 2011 /2012 season.

\begin{tabular}{|c|c|c|c|c|c|}
\hline \multirow{2}{*}{ Zones } & \multirow{2}{*}{ Governorates } & \multirow{2}{*}{$\begin{array}{l}\text { No. of } \\
\text { trials }\end{array}$} & \multirow{2}{*}{ Gemmeiz11 } & \multicolumn{2}{|c|}{ Checks } \\
\hline & & & & Giza 168 & Gemmeiza9 \\
\hline \multirow{4}{*}{ North Delta } & Damietta & 1 & 12.32 & 10.80 & 11.20 \\
\hline & \begin{tabular}{|l|} 
Kafr \\
El -Sheikh
\end{tabular} & 4 & 19.71 & 22.38 & 19.33 \\
\hline & Beheira & 2 & 24.86 & 20.86 & 21.28 \\
\hline & Alexandria & 2 & 22.40 & 22.26 & 22.68 \\
\hline Mean & & - & 19.82 & 19.07 & 18.62 \\
\hline \multirow{2}{*}{ Middle Delta } & Dakahlia & 2 & 25.65 & 21.75 & 23.80 \\
\hline & Gharbia & 2 & 28.00 & 22.23 & 30.00 \\
\hline Mean & & - & 26.83 & 21.99 & 26.90 \\
\hline \multirow{3}{*}{ South Delta } & Monufia & 3 & 23.80 & 24.12 & 20.81 \\
\hline & Qalyobia & 2 & 32.02 & 24.98 & 28.51 \\
\hline & Giza & 1 & 31.50 & 29.00 & 27.30 \\
\hline Mean & & - & 29.11 & 26.03 & 25.54 \\
\hline \multirow{4}{*}{ New Land } & Port Said & 1 & 21.77 & 17.86 & 22.93 \\
\hline & Ismailia & 1 & 25.90 & 21.01 & 24.50 \\
\hline & Suez & 1 & 20.16 & 17.64 & 17.92 \\
\hline & El-Bostan & 1 & 16.80 & 17.72 & 14.92 \\
\hline Mean & & - & 21.16 & 18.56 & 20.07 \\
\hline \multicolumn{2}{|l|}{ Zones mean } & 23 & 24.23 & 21.41 & 22.78 \\
\hline
\end{tabular}

Table (4-d): Average grain yield Ard/fed. of the newly released wheat cultivar Gemmeiza11 over all 66 verification yield trials in three growing seasons of 2009/2010, 2010/2011 and 2011/2012.

\begin{tabular}{|c|c|c|c|c|c|c|}
\hline \multirow[b]{2}{*}{ Zones } & \multirow[b]{2}{*}{$\begin{array}{l}\text { No.of } \\
\text { trials }\end{array}$} & \multirow[b]{2}{*}{$\begin{array}{l}\text { No.of } \\
\text { years }\end{array}$} & \multirow[b]{2}{*}{ Gemmeiza11 } & \multicolumn{3}{|c|}{ Checks } \\
\hline & & & & Sakha 93 & $\begin{array}{c}\text { Giza } \\
168\end{array}$ & Gemmeiza 9 \\
\hline North Delta & 17 & 3 & 19.52 & 17.91 & 19.28 & 18.74 \\
\hline Middle Delta & 16 & 3 & 22.21 & 17.98 & 19.03 & 20.48 \\
\hline South Delta & 16 & 3 & 26.37 & 24.44 & 25.12 & 24.92 \\
\hline Middle Egypt & 4 & 1 & 18.38 & 20.64 & 20.73 & - \\
\hline Upper Egypt & 4 & 1 & 18.17 & 18.41 & 18.90 & - \\
\hline New lands & 9 & 3 & 18.69 & 14.54 & 15.78 & 18.73 \\
\hline \multicolumn{3}{|l|}{ Grand mean } & 20.55 & 18.99 & 19.8 & 20.72 \\
\hline
\end{tabular}

\section{4- Demonstration fields}

Data in Table (5) show the average grain yield estimated for Gemmeiza 11 and the commercial wheat cultivars in 106 demonstration fields sown at 9 districts in 4 Governorates at Delta Region in 2011-2012 seasons. The results proved the superiority of the new cultivar Gemmeiza 11 over all the commercial wheat cultivars in the demonstration fields with an average of $(24.87 \mathrm{ard} / \mathrm{fad}$.) while, the neighboring fields recorded $(21.04 \mathrm{ard} / \mathrm{fed}$.) with $18.2 \%$ increment.

The results also proved the superiority of the new wheat cultivar Gemmeiza 11 at Delta region and the importance of planting on $120 \mathrm{~cm}$ width raised beds used in the demonstration fields compared to flat planting in the farmers' fields. 
Sadek, Eman M. et al.

5

196 
5-Stability parameters for grain yield:

Stability parameters for grain yield of the advanced yield trials in 2007/2008 growing season was calculated according to Eberhart and Russell(1966). The stable cultivar was defined as one which had a high average performance over a wide range of environments, a regression coefficient of 1.0 and no deviation from regression mean square. The results in Table 6 revealed that the new cultivar Gemmeiza 11 had better stability parameters at South Delta, Middle Egypt, Out Valley and at all over Egypt. Therefore, it could be recommended for planting in those environments.

Table (6): Grain yield stability parameters for Gemmeiza 11 along with the dominating wheat cultivars in 2007/2008 season.

\begin{tabular}{|c|c|c|c|c|}
\hline \multirow{2}{*}{ Region } & \multirow{2}{*}{ Cultivar } & \multirow{2}{*}{$\begin{array}{c}\text { Grain yield } \\
\text { (ard/fed) }\end{array}$} & \multicolumn{2}{|c|}{ Stability parameters } \\
\hline & & & $\mathbf{b}$ & $\mathrm{S}^{2} \mathrm{~d}$ \\
\hline \multirow{6}{*}{ North Delta } & Sakha 93 & 20.86 & 1.03 & 0.82 \\
\hline & Shandweel 1 & 21.56 & 1.12 & 3.32 \\
\hline & Gemmeiza 9 & 19.70 & 0.85 & 1.05 \\
\hline & Giza 168 & 20.02 & 0.98 & 0.30 \\
\hline & Checks mean & 20.48 & & \\
\hline & Gemmeiza 11 & 20.86 & 0.78 & 1.54 \\
\hline \multirow{7}{*}{ South Delta } & L.S.D.0.05 & 1.80 & 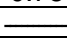 & - \\
\hline & Sakha 93 & 22.27 & 1.05 & 0.07 \\
\hline & Shandweel 1 & 23.45 & 1.24 & 29.36 \\
\hline & Gemmeiza 9 & 21.88 & 0.68 & 13.03 \\
\hline & Giza 168 & 23.01 & 0.80 & 16.51 \\
\hline & Checks mean & 22.65 & & \\
\hline & Gemmeiza 11 & 24.67 & 1.0 & 1.31 \\
\hline \multirow{8}{*}{ Middle Egypt } & L.S.D.0.05 & 1.45 & & \\
\hline & Sakha 93 & 24.77 & 1.05 & 0.43 \\
\hline & Shandweel 1 & 23.96 & 1.44 & 0.66 \\
\hline & Gemmeiza 9 & 24.15 & 1.32 & 0.68 \\
\hline & Giza 168 & 24.73 & 0.78 & 1.96 \\
\hline & Checks mean & 24.40 & & $\longrightarrow$ \\
\hline & Gemmeiza 11 & 27.13 & 0.95 & 0.15 \\
\hline & L.S.D.0.05 & 2.07 & & \\
\hline
\end{tabular}

Table (6) cont.

\begin{tabular}{|c|c|c|c|c|}
\hline \multirow{2}{*}{ Region } & \multirow{2}{*}{ Cultivar } & \multirow{2}{*}{$\begin{array}{l}\text { Grain yield } \\
\text { (ard/fed) }\end{array}$} & \multicolumn{2}{|c|}{ Stability parameters } \\
\hline & & & b & $\mathrm{S}^{2} \mathrm{~d}$ \\
\hline \multirow{6}{*}{ Upper Egypt } & Sakha 93 & 20.19 & 0.69 & 0.76 \\
\hline & Shandweel 1 & 23.35 & 1.20 & 0.55 \\
\hline & Gemmeiza 9 & 17.76 & 0.60 & -2.54 \\
\hline & Giza 168 & 19.97 & 1.11 & -2.27 \\
\hline & Checks mean & 20.07 & 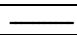 & - \\
\hline & Gemmeiza 11 & 21.32 & 1.75 & -4.40 \\
\hline \multirow{7}{*}{ Out Valley } & L.S.D.0.05 & 2.73 & & \\
\hline & Sakha 93 & 14.28 & 1.00 & 5.64 \\
\hline & Shandweel 1 & 15.10 & 0.83 & 1.29 \\
\hline & Gemmeiza 9 & 12.40 & 0.84 & 4.56 \\
\hline & Giza 168 & 15.48 & 0.93 & 6.57 \\
\hline & Checks mean & 14.31 & & \\
\hline & Gemmeiza 11 & 17.22 & 1.26 & 0.17 \\
\hline \multirow{8}{*}{ Over all Egypt } & L.S.D.0.05 & 1.02 & $\bar{L}$ & $\bar{C}$ \\
\hline & Sakha 93 & 20.97 & 1.06 & 0.49 \\
\hline & Shandweel 1 & 21.78 & 1.02 & 4.48 \\
\hline & Gemmeiza 9 & 19.86 & 0.92 & 6.25 \\
\hline & Giza 168 & 21.01 & 0.92 & 1.54 \\
\hline & Checks mean & 20.90 & $\bar{L}$ & $\overline{-}$ \\
\hline & Gemmeiza 11 & 22.90 & 1.06 & 1.50 \\
\hline & L.S.D.0.05 & 0.70 & - & $\longrightarrow$ \\
\hline
\end{tabular}




\section{6-Rust Disease Reaction:}

Data in Table 7 showed the average response of the bread wheat cultivar Gemmeiza 11 and four bread wheat cultivars to leaf and stem rust disease at Gemmeiza, Sakha and Nubaria Res. Stations in 2007/2008. The data revealed that Gemmeiza 11 was completely resistant to leaf rust at Gemmeiza Station and moderate susceptible at Sakha and Nubaria Stations (20Ms-30Ms). However, it was completely resistant to stem rust disease at the three stations. In the same time, the other cultivars showed different reactions to leaf and stem rust ranged from resistant to susceptible responses.

Table (7-a): Mean average of leaf and stem rust disease severity at adult stage for the new bread wheat cultivar Gemmeiza 11 and four wheat cultivars at Gemmeiza, Sakha and Nubaria Res. Stations in 2007/08 season.

\begin{tabular}{|l|c|c|c|c|c|c|}
\hline \multirow{2}{*}{ Cultivar } & \multicolumn{3}{c|}{ Leaf Rust } & \multicolumn{3}{c|}{ Stem Rust } \\
\cline { 2 - 7 } & Gemmeiza & Sakha & Nubaria & Gemmeiza & Sakha & Nubaria \\
\hline Gemmeiza 11 & 0 & $20 \mathrm{MS}$ & $30 \mathrm{MS} / \mathrm{S}$ & 0 & 0 & 0 \\
\hline Sakha 93 & $80 \mathrm{~s}$ & $60 \mathrm{~S}$ & $50 \mathrm{~S}$ & 0 & 0 & $20 \mathrm{~S}$ \\
\hline Gemmeiza 9 & 0 & 0 & $5 \mathrm{MS} / \mathrm{S}$ & 0 & 0 & $10 \mathrm{~S}$ \\
\hline Giza 168 & 0 & 0 & $20 \mathrm{MR} / \mathrm{MS}$ & 0 & 0 & $10 \mathrm{~S}$ \\
\hline Shandaweel 1 & 0 & 0 & $10 \mathrm{Mr}$ & 0 & 0 & 0 \\
\hline
\end{tabular}

Table (7-b): Mean of average coefficient of leaf and stem rust diseases infection at adult stage for the new bread wheat cultivar Gemmeiza 11 and four wheat cultivars at Gemmeiza, Sakha and Nubaria Res. Stations in $2007 / 08$ season.

\begin{tabular}{|l|c|c|c|c|c|c|}
\hline \multirow{2}{*}{ Cultivar } & \multicolumn{3}{|c|}{ Leaf Rust } & \multicolumn{3}{c|}{ Stem Rust } \\
\cline { 2 - 7 } & Gemmeiza & Sakha & Nubaria & Gemmeiza & Sakha & Nubaria \\
\hline Gemmeiza 11 & 0 & 16.00 & 24 & 0 & 0 & 0 \\
\hline Sakha 93 & 80 & 60 & 50 & 0 & 0 & 20 \\
\hline Gemmeiza 9 & 0 & 0 & 4.5 & 0 & 0 & 10 \\
\hline Giza 168 & 0 & 0 & 12 & 0 & 0 & 10 \\
\hline Shandaweel 1 & 0 & 0 & 4.00 & 0 & 0 & 0 \\
\hline
\end{tabular}

7- Distinctness Uniformity and Stability tests (DUS):

This test was carried out by the Central Administration of Seed Certification (CASC) for two successive seasons according to the International Union for the Protection of new Varieties of plants (UPOV).This test should be done before releasing the variety. Results of these tests are recorded in Table 8 showing some morphological characteristics of the new bread wheat cultivar Gemmieza 11. 
Table 8: Some morphological characteristics of the new released wheat cultivar Gemmeiza 11 according to the International Union for the Protection of new Varieties of plants (UPOV)

\begin{tabular}{|c|c|c|}
\hline No. & Characteristics & Description \\
\hline 1 & Pigmentation of coleoptile & Very weak (1) \\
\hline 2 & Anthocyanin coloration of first leaf & Very weak (1) \\
\hline 3 & Plant growth habit & Erect (1) \\
\hline 4 & Flag leaf rolling & Medium (5) \\
\hline 5 & Time of ear emergence(first spike on $50 \%$ of ears) & Early (3) \\
\hline 6 & Glaucosity of flag leaf sheath & Medium (5) \\
\hline 7 & Glaucosity of flag leaf blade & Medium (5) \\
\hline 8 & Ear: anthocyanin pigmentation & E \\
\hline 9 & Hairiness of upper most node & Weak (2) \\
\hline 10 & Glaucosity of ear neck & Strong (7) \\
\hline 11 & Glaucosity of ear & Mediun (5) \\
\hline 12 & Plant height (stem, ear and awns) & Long (7) \\
\hline 13 & Awns distribution on the spike & E \\
\hline 14 & Awns length compared to ear & Medium (5) \\
\hline 15 & Shape of lower glume & Elevated (7) \\
\hline 16 & Shoulder shape of glume & - \\
\hline 17 & Width of glume shoulder & Medium (5) \\
\hline 18 & Length of glume beak & Medium (5) \\
\hline 19 & Shape of glume beak & Medium (5) \\
\hline 20 & Hairiness of glume & - \\
\hline 21 & Thickness of parenchyma wall & Medium (5) \\
\hline 22 & Awns color & White (1) \\
\hline 23 & Ear length (ear and awns) & Long (7) \\
\hline 24 & Ear: hair density at the lower edge of the rachis & Weak (3) \\
\hline 25 & Color of ear & White (1) \\
\hline 26 & Shape of ear & Tapering (1) \\
\hline 27 & Density of ear & $\operatorname{Lax}(1)$ \\
\hline 28 & Grain shape & Elliptical (2) \\
\hline 29 & Grain color & White (1) \\
\hline 30 & Length of grain brush hairs & Short (3) \\
\hline 31 & Seasonal type & Spring type (3) \\
\hline
\end{tabular}

\section{8-Some technological and quality characters:}

Some technological and quality characters which are presented in Table 9 were carried out by Field Crops Technology Research Department, Food Technology Research Institute, ARC. The results showed that the cultivar Gemmieza11 had the lowest hectoliter value being 80.3 while the other five cultivars were higher in hectoliter weight and ranged from 81.0 to 83.8. This parameter is important for millers as it is positively correlated with the extraction rate (flour recovery). The extraction rate \% for Gemmeiza 11 was more than Gemmeiza 9 and Giza 168 and was equal to Sakha 94 cultivar.

Protein content is an important parameter for making different products of wheat flour. Protein content \% estimates of the new bread wheat cultivar Gemmeiza 11 along with the check cultivars Sandawell 1, Gemmeiza 9 and Giza 168 showed a high protein content $>12 \%$ which are suitable for making good quality bread. 
Gluten percentage of the six bread wheat cultivars which are presented in Table (9) showed that the gluten\% of Gemmeiza 9 was the highest $(30.8 \%)$ followed by Giza $168(28.8 \%)$, Sandaweel $1(26.9 \%)$, Sakha $94(26.5 \%)$ and Gemmeiza $11(24.3 \%)$ while Sakha 93 had the lowest value being (21.8\%).Similar rank was detected for dry gluten percentages where Gemmeiza 9, Sandaweel 1, Giza 168 and Gemmeiza 11 showed values of $10.3 \%, 9.7 \%, 9.1 \%$ and $9 \%$ respectively. Meanwhile, the two cultivars Sakha 93 and Sakha 94 recorded the lowest values of dry gluten being 7.2 and $8.5 \%$.

Table (9): Some technological and quality characters of the new bread wheat cultivar Gemmeiza 11and five commercial wheat cultivars.

\begin{tabular}{|l|c|c|c|c|c|c|}
\hline \multirow{2}{*}{ Cultivar } & $\begin{array}{c}\text { Hectoliter } \\
\text { weight } \\
\text { Kg. }\end{array}$ & Protein\% & Ash\% & $\begin{array}{c}\text { Extraction } \\
\text { Rate\% }\end{array}$ & \multicolumn{2}{c|}{ Gluten\% } \\
\cline { 5 - 7 } & & & & & Wet & Dry \\
\hline Sakha93 & 82.1 & 10.0 & 1.8 & 70.0 & 21.8 & 7.2 \\
\hline Sakha94 & 82.7 & 11.5 & 1.5 & 69.0 & 26.9 & 8.5 \\
\hline Shandaweel 1 & 83.8 & 12.5 & 1.9 & 71.3 & 26.5 & 9.7 \\
\hline Gemmieza9 & 81.0 & 12.6 & 1.7 & 68.5 & 3.08 & 10.3 \\
\hline Giza168 & 82.1 & 12.0 & 1.8 & 68.3 & 28.8 & 9.1 \\
\hline Checks mean & 82.3 & 11.7 & 1.7 & 69.4 & 26.9 & 8.96 \\
\hline Gemmeiza 11 & 80.3 & 12.2 & 1.7 & 69.0 & 24.3 & 9.0 \\
\hline
\end{tabular}

\section{9- Seed multiplication of the new cultivar Gemmeiza 11:}

In 2011 / 2012 growing seasons, Wheat Research Department produced a quantity of 671.6 ard. of the foundation seeds from the new cultivar and have been distributed either to the Central Administration of Seed Production or to the private seed companies to produced Register seeds.

Seeds of Gemmeiza 11 will be available for planting in farmers fields in 2013/ 2014 growing season. Foundation seeds will be maintained and distributed by Wheat Research Department, FCRI, ARC.Egypt.

It could be concluded that the new wheat cultivar Gemmeiza 11 can be grown at Delta region, Middle Egypt and Out Valley and can share the cultivar Shandaweel 1 at Upper Egypt to induce wide genetic variation which ensure higher degree of yield stability over any environmental fluctuations in the future.

\section{REFERENCES}

Abdel Shafi, A.M.; Gomma, A.A.; Khalil, O.H.S.; Enayat H. Ghanem, ElSayed, F.F.; El- Menoufi, M.M.; Mossad, M.G.; Gouda, M.A.; Mitkees, R.A.; El- Hadidi, M.M.; Abdel- Ghani, A.M.; Bassiouni, A.H.; Eissa, A.M.K.; Mahamoud, A.K.; Hanaa, N.S.; Abdel Aleem, M.M.; Shehab ElDin, T.M.; El-Shami, M.M.; Mahrous, M.A.; Sabry, S.R.S.; Mostafa, M.A.; El-Doudi, Y.H.; Ikhlas Shahik (1991): Gemmeiza 1 and Giza 165:two new bread wheat cultivars in Egypt. Assiut J. Agric. Sci. 22: 93 $-102$. 
Ageez, A.A.; Iman M.M. Sadek; M.S. Saleh and R.M. Mitkees. (1999).Genetic improvement in Egyptian wheat yield since 1950. J. Agric. Sci., Mansoura Univ., 24 (7): 3265-3278.

Eberhart, S.A. and W.A. Russell. (1966): Stability parameters for comparing varieties. Crop Sci., 6:36-40.

El-Sayed, F.F.; Ali, A.M.A.S.; El.Menoufi, M.M.; Mitkees, R.A.;Hamada, A.A.; Mahrous, M.A.; Shehab El Din, T.M.; El Shami, M.M.; Mostafa, M.A.; Abdel- Ghani, A.M.; Eman M.M. Sadek;Ali, A.M.; Iskandar, M.H.; Bassiouni, A.H.; Eissa, A.M.K.; Abdel-Gawad, Y.G.; Kadria Hegazi and Y.H. El-Doudi, (1996): An Introduction to the new bread wheat cultivar Gemmeiza 3. J. Agric.Sci. Mansoura Univ.; 21 (11) 3811-3823, 1996.

El Shami, M.; T. Shehab El Din; M. Mostafa; M. Abdel Aleem,; M .Mahrous ; A. Ageez; A.Hamada; A. Bassiouni; M. Eid; A. Abdel- Ghani; M. Eskandar; S.Sabry; M. Sharshar; Iman Sadek; A. Abo- Warda; A.M. Mousa; E. El-Sayed ; H. Ashoush; M. Towfeeles; Hayam Mahjoub; A. Moustafa; H. Hendawy; F. Hefnawy; S. Ali; A. Abdel-Karim; A. Menshawy; H. El-Borhamy; M. Abdel- Fattah; G.A. El- Shaaray; S. ElSawi; R. Kumber; Sabah. Abo Alela; Waffa. El- Awady; I. Amin; A.Moussa; S. Abdel Dayem; M. Zakreia; S. Hammad; A. Swaliem; A. Gomaa; O. Khalil; Kadria Hegazi; Enayat Khanem; R. Mitkees; M. ElMonofy; A.M. Mousa; A. Abdel- Latif; N.Hanaa; A.Khattab and M. El Shami. (2005): Gemmeiza 10: A New Egyptian High Yielding and Rust Resistant Bread Wheat Cultivar, J.Agric. Sci., Mansoura Univ.; 30:743754.

Ghanem, Enayat H.; Abdel- Shafi Ali A.M.; Hanna, N.S.; Mahmoud, S.K.; Sabry, S.R.S.; Abdel-Aleem, M.M.; Zaid, H.M.M.; Moussa, A.M.; Abdel Majed, S.A. and S.Sherif, (1996): Grain yield and stability of the new bread wheat Triticum aestivum L, cultivar Sids 1 in different agroclimatic zones of Egypt. Bull. Fac. Agric., Univ. Cairo, 47 (4): 565676.

Gomaa, A.S.; Abo- Elenein. R.A.; Dessouki, S.M.; Sadek, M.M.; Talaat, E.H.; Khalil, O.H.; Attia, S.A.; Kadria F, Hegazi; Enayat H, Ghanem and M.A. Gouda, (1979): Giza 157 and Sakha 8: two new bread wheat varieties. First General conferance of ARC, Giza, Egypt, May 22-29.

Gomaa, A.S.; Khalil,O.S.; Abo Elenein. R.A.; Kadria F, Hegazi; Enayat H, Ghanem; Shafi Al, A.; El- Sayed, F.F.; Gouda, M.A. El Shami, M.; Ageez, A.A.; Saleh, M.E.; El- Hadidi, M.; Atta, S. and M.G.Mosaad (1984). Sakha 69 and Sakha 61: two new bread wheat high- yielding varieties. Second General Conference of ARC, Giza, Egypt, April $9-11$.

Mahrous, A.M.; M.Abdel- Aleem; T. Shehab El- Din; M. Mostafa;S. AbdelMajeed; S.Sabry; Iman M. Sadek; M.Sharshar; A. Hamada; A. AboWarda;A.M. Moussa; A. Tammam; M.Meshref; E.El-Sayed; H. Ashoush; M. Toweefles; H. Hendawy; Hayam S. Mahgoub; A. Mostafa; H. El-Borhamy;A.Menshawy; A.Moussa; Wafaa M. El-Awady; A.ElHag; R.Koumbor; S.Seleem; R.A.Ramadan; Nadia A.Abdel- Nour; G. Sharawy; Sohair M.Hassan; A. Sewelam; S. El- Saway; S. AbdelDayem; A. El-Sebaey; Magda A. Abdel-Rahman; Sabah. Abo 
Alela;M.Khaled; I.Amin; M. Zakaria; Manal A. Hassan; A. Gad-Allah; S. Hammada;M.El-Maghraby; A. Morad; Aza, M.Abdel -All; A. Hagras; A.T. Moustafa; M.Mahoud; M.Moubarak; Kadria Hegazi; A.Gomaa; O.Khalil; Enayat Khanem; R. Meetiks; M. El- Monofy; S. Kh. Mahmoud; N.Hanna; M.Eid; M.Mosaad;M.A.Gouds; A.Ageez; M.Abdel-Fattah; A.Khattab; A. A.Abdel-Lattif; M.Eskander; Najwa Abdel- Fattah; F. Hefnawy and W. Abdel- Samad.(2009).Grain yield and Stability of the new Egyptian bread wheat cultivar Sids 12. J.Agric. Sci.,Mansoura Univ., 34(4): 3199-3209.

Mitkees, R.A.; M.A. El-Monofy; A.A. Hamada.; F.F. El- Sayed.; A.A. Ageez; M.A. Mahrous; H. Ashoush; A.A Gomaa.; O.H.S Khalil.; Kadria F, Hegazi; Enayat H, Ghanem; A.A. Ali; M.G. Mosaad; M.M. El-Shami; A.H. Bassiouni; A.M.K. Eissa; T.M. Shehab El Din; M.M. Abde-Aleem; S.Kh. Mahmoud; M.A.M Eid; M.A. Mostafa; M.H. Iskandar; N.S. Hanna; S.R.S. Sabry; A.H. Abdel- Latif; A.M.A. Abo- Warda; Y.G. AbdelGwad; A.M. Mousa; S.A. Abdel- Majeed; H.M. Zaid; A.M. Tammam; Nagwa R. Abdel- Fatah; M.Kh. Mosherf; E.A.M. El-Sayed; Hayam S. Mahgoub; M.B. Toweefles; S. Abdel- Halim; A.K. Mostafa; F.A. Hefnawy; Y.H. El-Daoudi; M.O. Khilifa and M.M. El- Shamy(1998). Gemmeiza 5: A new Egyptian bread wheat cultivar. Annals of Agric. Sci.,Moshtohor, $36:$ : 1) 43-59.

Mosaad M.; M. El- Monofy; T. Shehab El- Din; R.Mitkees; M. Mahrous; A. Hamada; A.Ageez; A. Bassiouni; M. El-Shami; M.Abdel-Aleem;M. Eid; A. Abdel- Ghani; M.Eskander; N. Hanaa;S.Sabry; A. Abdel-Latif; M.Sharshr; Iman Sadek; M.Mostafa;A.Abo-Warda; Y.Abdel-Gwad; A.Mousa;S.Abdel-Majeed;A.Tammam;NajwaAbdel-

Fattah;M.Moshref;E.El-Sayed; H.Ashoush; M.Towfeeles; Hayam Mahjoub; A.Moustafa; F.Hefnawy; H. Hendawy; S.Ali; A.Abdel-Karim;

A. Khattab; M.Abdel-Fattah; A. Menshawy; H.El-Borhamy; A.Gomaa;F.

O. Khalil; Kadria Hegazi; A.Ali; Enayat Khanem; S.Mahmoud and M.Khalifa(2000). Gemmeiza 9: A new Egyptian high yielding and rust resistant bread wheat cultivar for Delta region. J.Agric. Sci.,Mansoura Univ.,25 (12): 7407-7419.

Moustafa, M.A.; M.S.Sharshr; T. Shehab El- Din; M.Abo- Shereef; S.AbdelMajeed; M.Abdel -Aleem; S.R.S.Sabry; Iman M. Sadek; A. Hamada; A.Abo-Warda; A.Tammam; M.Moshref; E. El-Sayed; H.Ashoush; M.Towfeeles; H. Hendawy; Hayam S. Mahjoub; A.K. Moustafa; H.ElBorhamy; A. Menshawy; A.Moussa; Wafaa M. El-Awady; A. El-Hag; R.Koumbor; S. Seleem; R.A. Ramadan; Nadia A. Abdel- Nour; G.Sharawy; S. Abdel- Dayem; Sohair M. Hassan; A. Sewelam; S.ElSawy; S. Hammad; Magda A. Abdel- Rahman; S. Abo- Elela; M.A. Khaled; I.A. Amin; M. Zakaria; Manal A. Hassan; A. Gad- Allah; M.A. El- Maghraby; A.Morad; Aza M. Abdel- Al; A. Hagras; A. T. Moustafa; M. S. Mahmoud; M. Y. Mubark; Hoda M. M El- Gharbawy; A.A. Mahmoud; A. Gomaa; Enayat Khanem; R. Mitkees; M.El- Monofy; S.Kh. Mahmoud; N. Hanna; M.A. Moussa; M.A. Gouda; A. Ageez;M. A. Salem; A. Khattab; A. Abdel-Latif; M. Eskander; NajwaAbdel-Fattah; F. 
Hefnawy and W. Abdel- Samad(2010).Sids 13: A new bread wheat cultivar. J. of Plant Production,Mansoura Univ1 (1): 147-157.

Shehab El-Din T.; R.A. Mitkees.; M.M. El- Shamy; M.A. Gouda; M.M. AbdeAleem; A.M. Abdel- Ghani; N.S. Hanna; Iman M.M. Sadek; A.M. AboWarda; M.Kh. Mosherf; E.A.M. El-Sayed; Hayam S. Mahgoub; A.K.Mostafa; M.G. Mosaad; A.H. Bassiouni; M.M.A. El-Monofy; S.Kh. Mahmoud; M.A. Mahrous; A.A. Ageez; M.A.M Eid; M.H. Iskandar; M.A. Mostafa; A.A. Hamada; Y.G. Abdel- Gwad; A.M. Mousa; S.A. AbdelMajeed; A.M. Tammam; Nagwa R. Abdel- Fatah; H. Ashoush; F.A. Hefnawy; H. Hendawy; S.El-Din Ali; M.B. Toweefles; A.A. Abdel-Karim; A.A.Khattab; A.A.Gomaa;O.H.S.Khalill; Kadria F, Hegazi; Enayat $H$, Ghanem; A.A.Ali; F.F. El-Sayed; Ikhlas Safik and S. AboNaga,(1999):Sakha 93 and Giza 168:Two new high yielding and rust diseases resistant bread wheat cultivars. j. Agric. Sci., Mansoura Univ., 24:2157-2168.

Shehab El-Din T.; M. El- Shami; A. Abdel-Latif; M. Mostafa; M. Abde-Aleem; M. Sharshar; A. Menshawy; H. El-Borhamy; S.Hammad; M. Mahrous; A. Ageez; A. Bassiouni; M.Eid; A. Abdel- Ghani; M. Eskandar; S. Sabry; Iman Sadek; A. Hamada; A. Abo- Warda; S. Abdel- Majeed; A.Tammam; Nagwa Abdel- Fatah; M.Mosherf; E.El-Sayed; H. Ashoush; M.Toweefles; Hayam Mahgoub; A. Moustafa; F. Hefnawy; $\mathrm{H}$. Hendawy; S. Ali; A. Abdel-Karim; M. Abdel- Fatah; G.El- Shaaray; S. El- Sawi; R. Kumber; Sabah. Abo Alela; Waffa. El- Awady ; I.Amin; A.Mousa; S. Abdel-Majeed; A.M. Mousa; S Abdel Dayem; M. Zakeria; A.Swailem; A. Gomaa;O.S Khalil.; Kadria F, Hegazi; Enayat $\mathrm{H}$, Ghanem; R. Mitkees, M. El-Monofy; N.Hanna; A.Khattab and W. Youssof(2005):An Introduction to Sakha 94, The new bread wheat cultivar. J.Agric.Sci., Mansoura Univ., 30:91-101.

Steel. R.G.D. and Torrie, J.H (1960): Principles and procedures of Statistics. $2^{\text {nd }}$ Ed. Mc Graw-Hill Book Co. New York. 
جميزة 11 : صنف قمح خبز جديد عالى المحصول

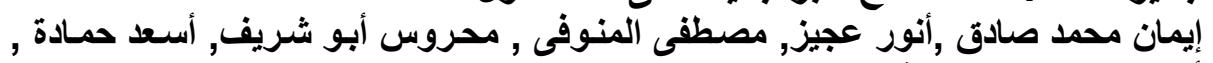

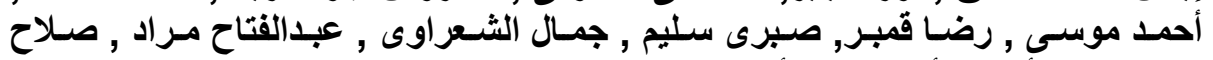

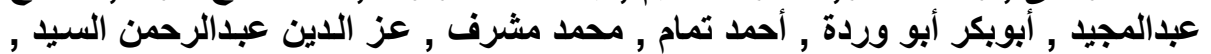

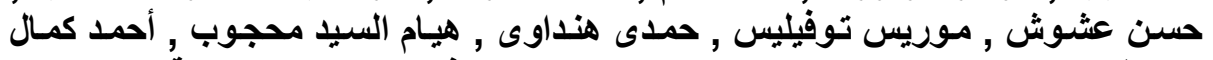

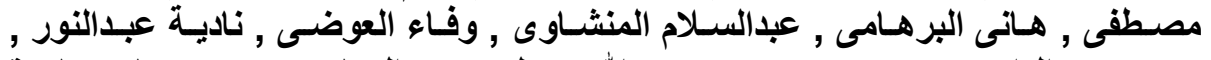

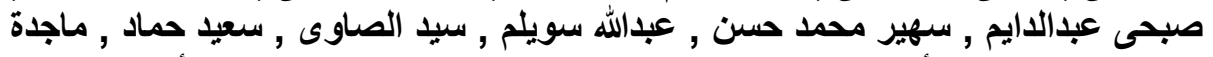

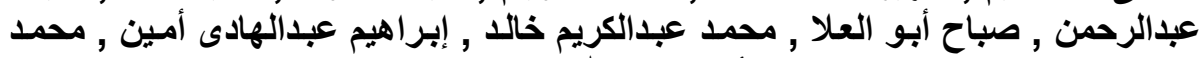

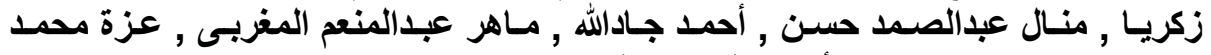

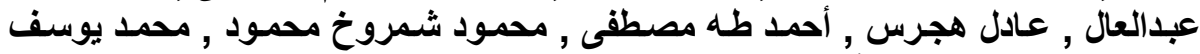

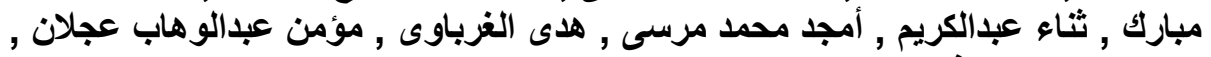

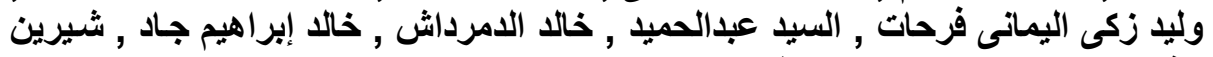

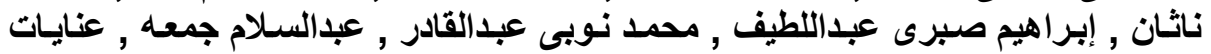

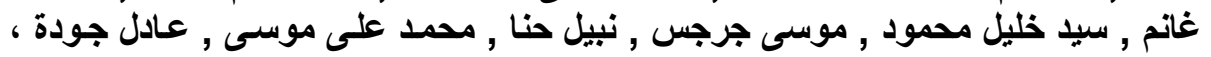

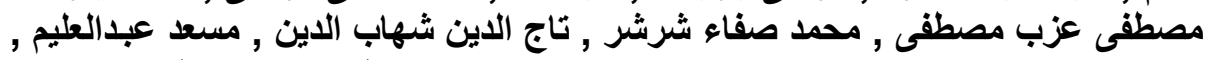

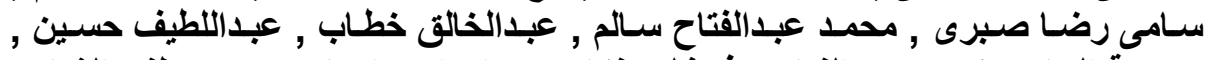

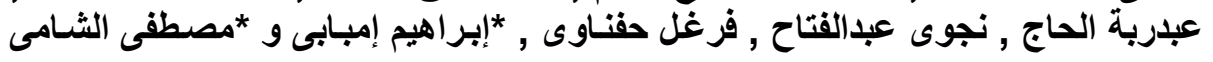

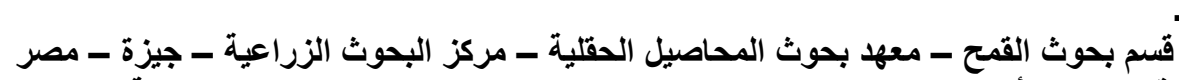

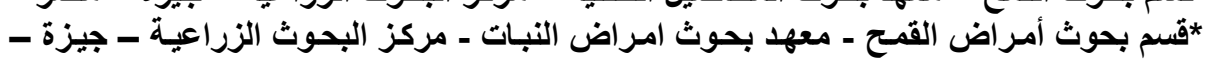

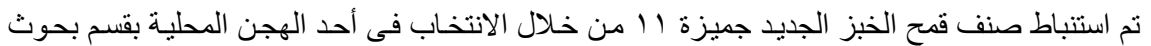

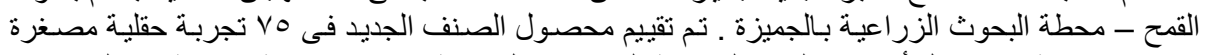

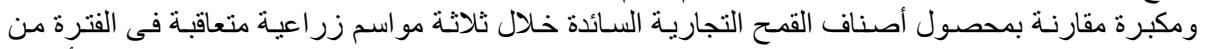

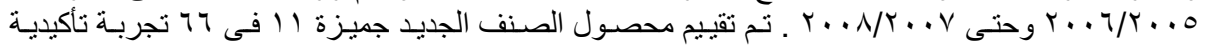

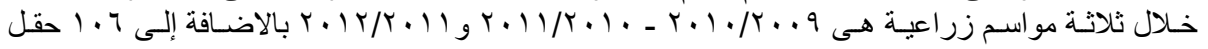

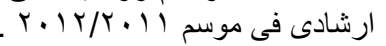

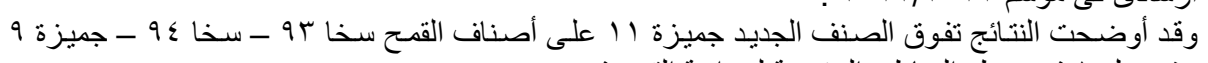

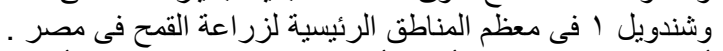

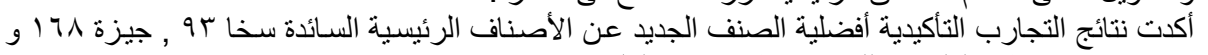

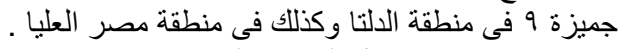

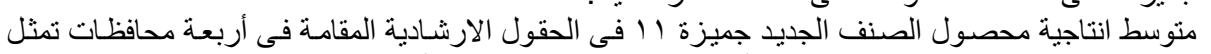

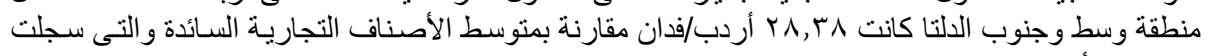

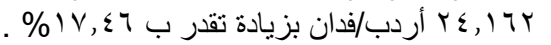

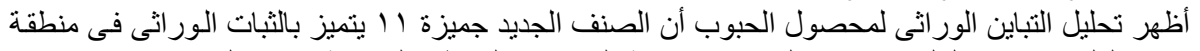

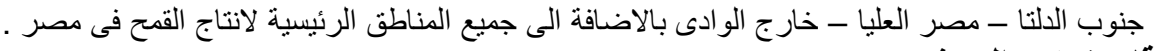

كلية الزراعة - جامعة المنصورة كلية الزراعة - جامعة الزعة جامعة المنصورة قام بتحكيم البحث أ.د / أدمد نادر السيد عطيه أ.د / احمد محمد عبد الغنى نادر الـيد عطيه 
J. Plant Production, Mansoura Univ., Vol. 4 (2), February, 2013 

Table (5): Average grain yield (Ard/fad) of the new released cultivar Gemmeiza 11 and 5 commercial wheat cultivars in and out demonstration fields in 2011/2012 season.

\begin{tabular}{|c|c|c|c|c|c|c|c|c|c|c|c|c|c|c|}
\hline \multirow[b]{3}{*}{ 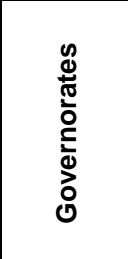 } & \multirow[b]{3}{*}{$\begin{array}{l}\frac{0}{0} \\
\frac{0}{2} \\
\frac{\omega}{0} \\
\frac{0}{0}\end{array}$} & \multicolumn{12}{|c|}{ Cultivars } & \multirow[b]{3}{*}{ 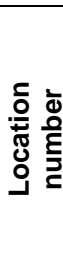 } \\
\hline & & \multicolumn{2}{|c|}{ Gem. 11} & \multicolumn{2}{|c|}{ Gem. 9} & \multicolumn{2}{|c|}{ Sids 12} & \multicolumn{2}{|c|}{ Giza 168} & \multicolumn{2}{|c|}{ Misr 1} & \multicolumn{2}{|c|}{ Misr 2} & \\
\hline & & 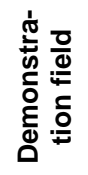 & 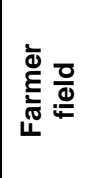 & 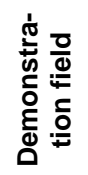 & 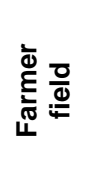 & 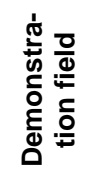 & 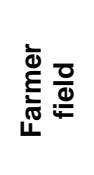 & 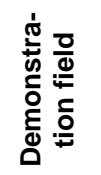 & 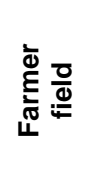 & 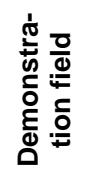 & 离 & 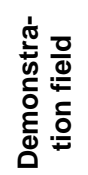 & 离 & \\
\hline \multirow{2}{*}{ Gharbia } & $\begin{array}{l}\text { Mehalla El- } \\
\text { Kobra }\end{array}$ & $\begin{array}{c}30.18 \\
(1) \\
\end{array}$ & 19.00 & $\begin{array}{c}25.75 \\
(2) \\
\end{array}$ & 22.25 & $\begin{array}{l}24.5 \\
(2) \\
\end{array}$ & 21.00 & $\begin{array}{l}24 \\
(1)\end{array}$ & 19.00 & $\begin{array}{r}27.8 \\
(2) \\
\end{array}$ & 18.5 & - & - & 8 \\
\hline & El-Santa & $\begin{array}{c}29.55 \\
(1)\end{array}$ & 23.00 & - & - & $\begin{array}{c}25.57 \\
(2)\end{array}$ & 21.1 & $\begin{array}{c}19.00 \\
(1)\end{array}$ & 18.00 & $\begin{array}{c}22.85 \\
(2)\end{array}$ & 18.62 & - & - & 6 \\
\hline \multirow{4}{*}{$\begin{array}{l}\text { El- } \\
\text { Sharkia }\end{array}$} & Zagazig & $\begin{array}{c}30.60 \\
(1)\end{array}$ & 26.10 & - & - & $\begin{array}{c}25.06 \\
(8)\end{array}$ & 20.15 & $\begin{array}{r}23.4 \\
(4) \\
\end{array}$ & 19.12 & $\begin{array}{c}24.48 \\
(8) \\
\end{array}$ & 19.7 & $\begin{array}{r}24.61 \\
(3) \\
\end{array}$ & 19.76 & 24 \\
\hline & \begin{tabular}{|l|} 
Menya \\
Alkamh \\
\end{tabular} & $\begin{array}{c}24.75 \\
(1) \\
\end{array}$ & 22 & - & - & $\begin{array}{l}25.1 \\
(7) \\
\end{array}$ & 21.14 & $\begin{array}{c}20.75 \\
(2) \\
\end{array}$ & 19.25 & $\begin{array}{c}23.29 \\
(9) \\
\end{array}$ & 19.74 & $\begin{array}{c}25.77 \\
(2) \\
\end{array}$ & 21.5 & 21 \\
\hline & Abo-Kbeer & $\begin{array}{c}25.30 \\
(1)\end{array}$ & 20.65 & - & - & $\begin{array}{c}23.35 \\
(5)\end{array}$ & 19.01 & $\begin{array}{c}22.12 \\
(1)\end{array}$ & 18.1 & $\begin{array}{r}22.14 \\
(1)\end{array}$ & 19.10 & $\begin{array}{c}20.06 \\
(2)\end{array}$ & 18.82 & 10 \\
\hline & Fakous & $\begin{array}{c}24.30 \\
(1)\end{array}$ & 20.30 & - & - & $\begin{array}{c}22.64 \\
(5)\end{array}$ & 19.57 & $\begin{array}{c}22.52 \\
(2)\end{array}$ & 18.93 & $\begin{array}{c}22.32 \\
(7)\end{array}$ & 19.25 & $\begin{array}{c}24.83 \\
(2)\end{array}$ & 18 & 17 \\
\hline \multirow{2}{*}{$\begin{array}{l}\text { El- } \\
\text { Menofiya }\end{array}$} & \begin{tabular}{|l|} 
El- \\
Shohadaa
\end{tabular} & $\begin{array}{c}28.85 \\
(1)\end{array}$ & 25 & - & - & $\begin{array}{c}29.00 \\
(2)\end{array}$ & 26.72 & - & - & $\begin{array}{c}26.75 \\
(2)\end{array}$ & 24.47 & - & - & 5 \\
\hline & $\begin{array}{l}\text { Shebeen } \\
\text { El-Kom }\end{array}$ & $\begin{array}{c}31.92 \\
(1)\end{array}$ & 25 & $\begin{array}{c}22.54 \\
(2) \\
\end{array}$ & 20.62 & $\begin{array}{c}26.27 \\
(3) \\
\end{array}$ & 23.66 & - & - & $\begin{array}{c}23.35 \\
(1) \\
\end{array}$ & 21.15 & $\begin{array}{c}24.70 \\
(1) \\
\end{array}$ & 23 & 8 \\
\hline \begin{tabular}{|l|} 
El- \\
Dakhalia
\end{tabular} & Talkha & $\begin{array}{c}30.02 \\
(1)\end{array}$ & 25.50 & - & - & $\begin{array}{c}26.98 \\
(2)\end{array}$ & 21.9 & $\begin{array}{r}24.9 \\
(1)\end{array}$ & 22 & $\begin{array}{r}27.58 \\
(2)\end{array}$ & 23.85 & $\begin{array}{r}26.87 \\
(1)\end{array}$ & 22.4 & 7 \\
\hline Mean & & 28.38 & 22.95 & 24.14 & 21.43 & 25.38 & 21.58 & 22.32 & 19.2 & 24.5 & 20.48 & 24.47 & 20.58 & 106 \\
\hline
\end{tabular}

Mean of demonstration fields $=24.865 \mathrm{ard} / \mathrm{fed}$

Mean of five check cultivars in demonstration fields $=24.162 \mathrm{ard} / \mathrm{fed}$

Mean of farmer fields $=21.037 \mathrm{ard} / \mathrm{fed}$

Grain yield incerement $=3.828 \mathrm{ard} / \mathrm{fed}(18.20 \%)$

Mean of five check cultivars out demonstration fields $=20.654 \mathrm{ard} / \mathrm{fed}$

Average increamnt of Gemmeiza 11compared to five commercial wheat cultivars in demonstration fields $=4.218$ ard $/$ fed $(17.46 \%)$ 